\title{
Reduction of Contact Stress by Use of Relief Notches
}

\author{
by M. Bijak-Zochowski, A.M. Waas, W.J. Anderson and C.E. Miniatt
}

ABSTRACT-The photoelastic method is used to investigate the possibility of relieving the large local stresses that develop in the corners of a right angled indenter compressing a semiinfinite body by inducing geometric changes to the indenter/ semi-infinite body configuration. It is shown that a circular notch cut along the free edges of the indenter can totally eliminate the large corner stresses. The notch, if placed along the interface edge of the half plane, can reduce the stress concentration, but never eliminate it. The results obtained have wide practical application.

\section{Introduction}

It is well known in contact problems that a stress singularity exists at the sharp corner of a wedge indenter compressing a semi-infinite body. The power of the singularity depends on the angle of the wedge and on the friction between the indenter and the body. ${ }^{1-5}$ The investigations reported here describe how the singularity (stress concentration) can be controlled and eliminated by relief cuts (notches) either along the free edges of the indenter (Fig. 1) or along the interface edge of the half-plane (Fig. 2). An indenter with right angle corners is investigated.

Research on this problem is motivated by the difficulties associated with the design of the upper end of an engine connecting rod, where the rod is shrunk on the wrist pin. A related, purely axisymmetric case, is that of shrink fit of a turbine disk on a shaft. The use of notches (circular grooves) in the shaft as a stress relief device is known in the steam turbine industry. ${ }^{6}$ Although the current tests are done with plane-stress conditions, the results shed light on the related axisymmetric case.

\section{Experimental Procedure}

The photoelastic method was used. The specimens and the 'half plane' were cut from a $3 / 8$-in. thick plate of PSM-1 polycarbonate plastic ( $f$ value, $c=40 \mathrm{psi} /$ fringe in.). The general dimensions of the specimens and of the models of half-planes are shown in Figs. 1 and 2. The interface was lubricated with a light oil to diminish the friction at the contact surface, but not to change the pressure distribution. (An oil film or grease can change pressure distribution, depending on the kinematic viscosity.) The friction was not totally eliminated, however.

The interface edges of the 'notched-indenter' set of specimens (Fig. 1) were finished carefully by fine milling. As the experimental work progressed, it was felt that the specimen preparation technique could be improved. The interface edges of the 'notched half-plane' set of specimens (Fig. 2) were first finished by fine milling and thereafter polished using very fine sand paper. This latter set of

M. Bijak-Zochowski is Professor, The Technical University of Warsaw, Poland. A.M. Waas is Assistant Professor, W.J. Anderson is Professor, and E.C. Minialt is Graduate Student, The Department of Aerospace Engineering, The University of Michigan, Patterson Place, Ann Arbor, MI 48109-2140.

Original manuscript submitted: October 17, 1990. Final manuscript received: June $25,1991$. specimens yielded a smoother contact; the quality of contact was ascertained by inspecting the photoelastic fringe patterns in the vicinity of the contact surface.

The notched indenter set consisted of 22 specimens, each with different distance $l$ between the circular notch (1-in. diameter) and the indenter sharp corner. The nondimensional distance $\eta=l / d$ ranged from 1.10 to 4.00 (Fig. 1). The dimensions $w_{i}=4$ in., $w_{h}=8$ in. and $h=$ 5 in.

The notched half-plane set consisted of three half-plane configurations with different notch radii $(0.5,0.25$ and $0.125 \mathrm{in}$.) and many different width indenters. The nondimensional distance $\zeta=L / D$, ranged from 0.50 to 3.50 (Fig. 2). The dimensions $a=4$ in., $w_{h}=8$ in. and $H=4$ in. The average pressure applied to the indenter was held constant at $q=550$ psi.

Pictures of the isochromatic fringe pattern were taken by a Nikon F3 camera with 105-mm Micro-Nikkor lens. The nondimensional radial distance $\bar{r}=r / r_{0}$ between the sharp corner and a particular fringe was measured for a 70-deg azimuthal angle from the contact surface. The reference distance $r_{0}$ was 0.040 in. $(1 \mathrm{~mm})$. Each frame of the film was magnified to get a $1: 24$ enlargement of the image.

\section{Results and Analysis}

The results of the investigations for the 'notchedindenter' case are shown in Figs. 3-5. Each data point represented in Figs. 3 and 5 is the average for the two corners of the indenter for each test.

Plotting $\ln$ (fringe order) versus $\ln (\bar{r})$, one obtains straight lines for power stress singularities. This follows from the analysis presented in Dundurs ${ }^{1}$ (for an unnotched situation) where it is shown that the stresses vary according to

$$
\sigma=A r^{p-1}
$$

In some cases however, the relations were clearly nonlinear, especially for very small $\bar{r}$. Such results are due to different reasons: in a small corner region, the material can behave inelastically; the error of the measurements increases dramatically for small $\bar{r}$; the error of specimen machining plays a much greater role in close vicinity of the sharp corner and-in general-the stress singularity for an indenter with notches may not be exactly of the 'power' type. In each case, however, the plots of In (fringe order) versus $\ln (\bar{r})$, revealed at least four data points (always more than half) that fell along a straight line. It was decided, therefore, to describe the singular stress field by eq (1).

The $p$ value that is related to the power of the singularity can be found by calculating the slope of the $\ln$ (fringe order) versus $\ln (\bar{r})$ straight line according to the equation

$$
C+\ln (\text { fringe order })=(p-1) \ln \bar{r}+D
$$

where $\bar{r}$ is the nondimensional distance mentioned before, $C$ and $D$ are constants. 
Figure 3 shows the effect on $p$ of the distance between the notch and the corner (described by $\eta$ ). In this figure, results are presented for two different sets of tests and a numerical calculation performed using the finite-element method. Details of the numerical work are reported in Ref. 7 and elaborations of the results corresponding to test set $A$ are reported in Ref. 8. An asymptote corresponding to the situation without a notch in the indenter is also indicated in this figure. The corresponding analysis is given in Ref. 1. All the results in this figure indicate

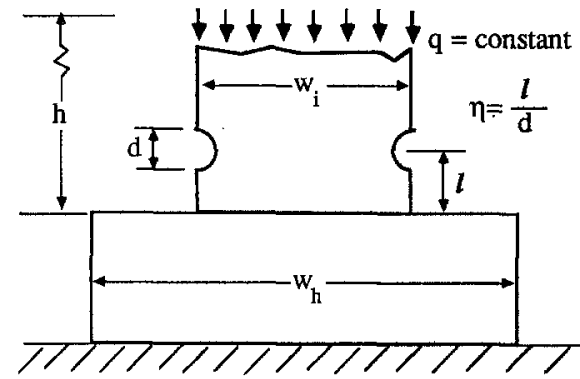

Fig. 1-Notch in indenter

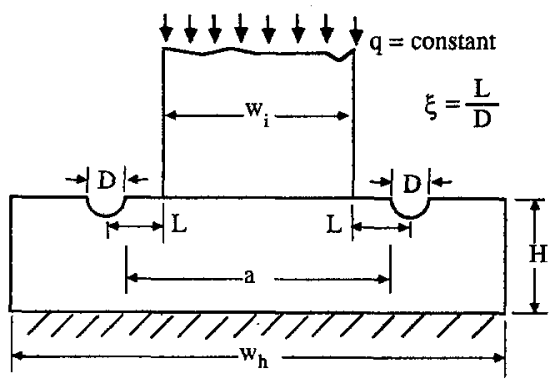

Fig. 2-Notch in half plane

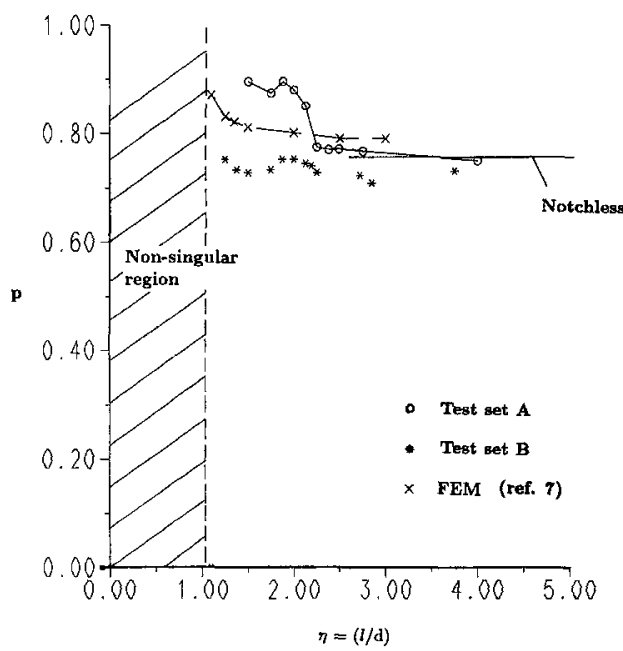

Fig. 3-Strength of singularity as a function of $\eta$, for notch in indenter that, as the notch approaches the corner the relation (1) seems not to hold. This is because the relation between $\ln$ (fringe order) and $\ln (\bar{r})$ deviates from being linear for values of $\eta$ less than approximately one. This region is marked 'nonsingular' in Fig. 3. Both sets of experimental data appear to have captured the trend in the variation of $p$ against $\eta$ when compared with the FEM result. The important point to note here is that all of the results point to the disappearance of the singular stress state (1) for small values of $\eta$.

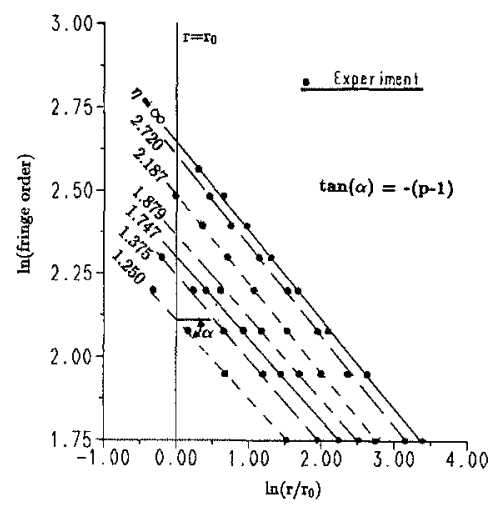

Fig. 4-Variation of In (fringe order) as a function of $\ln (\bar{r})$ for different $\eta$. Notch in indenter

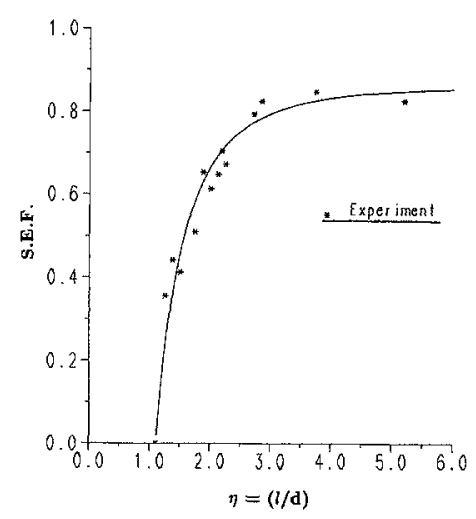

Fig. 5(a)-Stress excess factor in presence of notch relief. Notch in indenter.

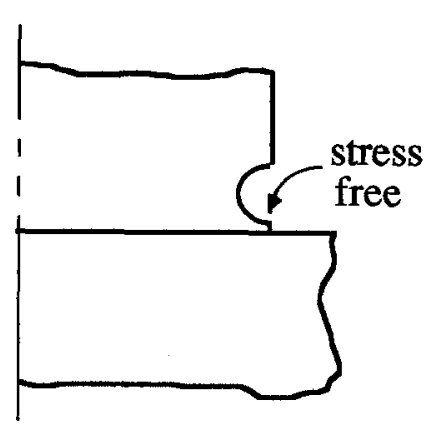

Fig. 5(b)-Sketch of stress-free material at corner 


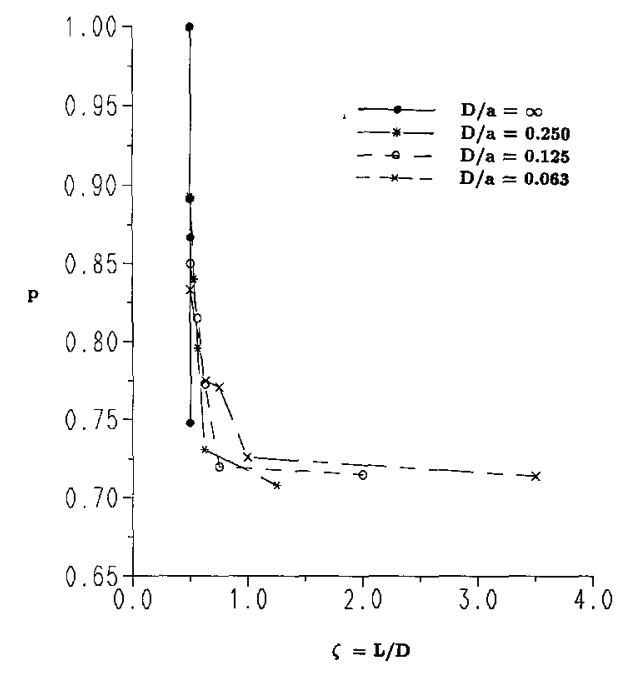

Fig. 6(a)-Relations between stress singularity power $(p)$ and nondimensional distance $\zeta$. Notch in half plane
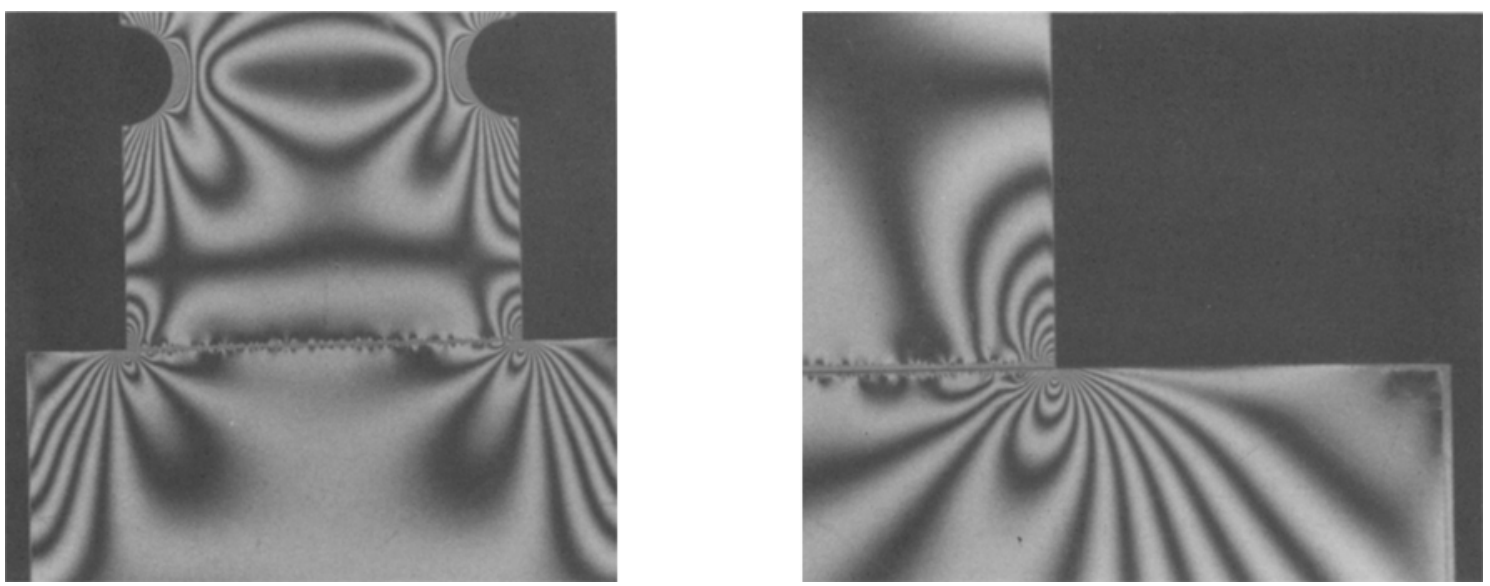

(a)
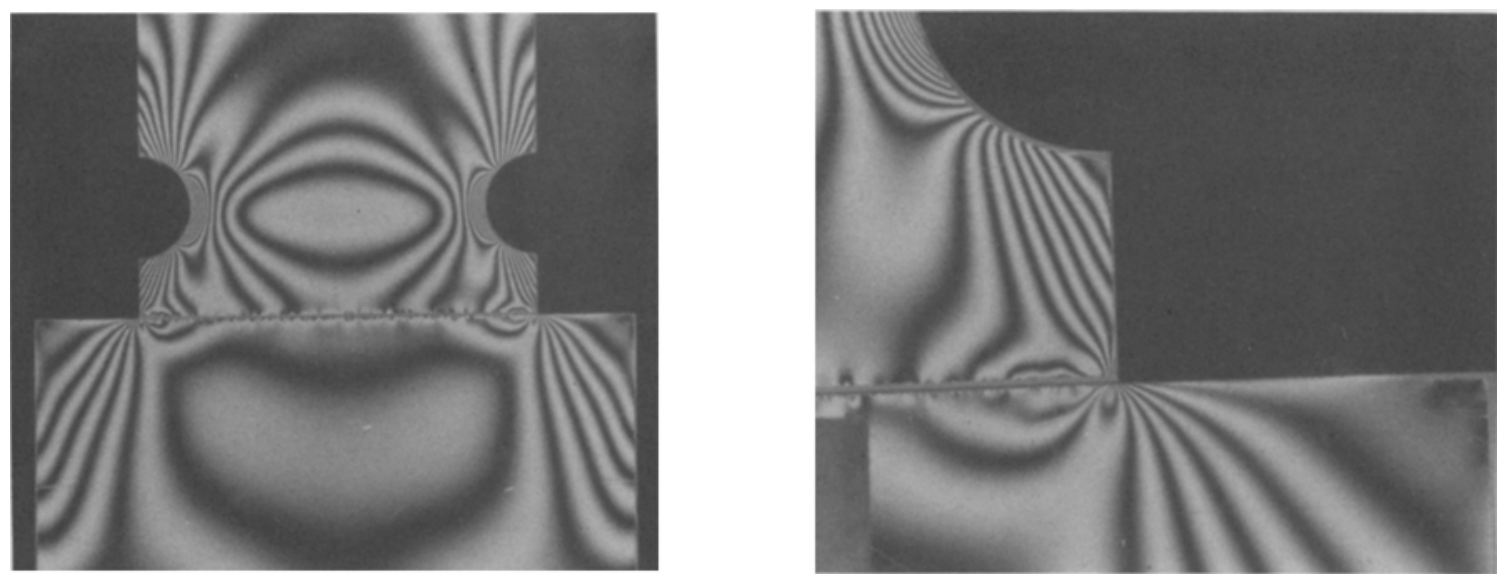

(b)

Fig. 7-Fringe patterns for the 'notch in the indenter' case. (a) $\eta=2.72$ : total picture and the enlargement of the right corner. (b) $\eta=1.10$ : total picture and the enlargement of the right corner 
Plots of $\ln$ (fringe order) versus $\ln (\bar{r})$ for different $\eta$ resulted in straight lines parallel to each other as shown in Fig. 4. The stress-excess factor, $*$ SEF $=\ln$ (fringe order $/ 6$ ) can be evaluated in each case as a vertical distance between a particular line and the $\ln (6)$ value. The fringe order $=6$ was the lowest fringe order in the corner, a little exceeding that corresponding to the average pressure $q=550$ psi. The stress-excess factor was evaluated for

${ }^{*}$ SEF $=S C F-1$, where $S C F$ stands for the usual definition of stressconcentration factor $r=0.040 \mathrm{in} .(1 \mathrm{~mm})$. It was assumed that if the $6^{\text {th }}$ fringe order appears at the distance $r=1 \mathrm{~mm}$ the stress excess vanishes.

The relation between the stress-excess factor and the nondimensional notch distance $\eta$ is shown in Fig. 5(a). The points indicate experimental data, and the curve was plotted using the minimum square-root deviation method. The stress excess disappears for values of $\eta \leq 1.1$ which indicates that the full relief in the corners was attained for values of $\eta$ in this range. This result is not unexpected because as the notch gets closer to corners a strip of material at the corners is almost stress free due to the
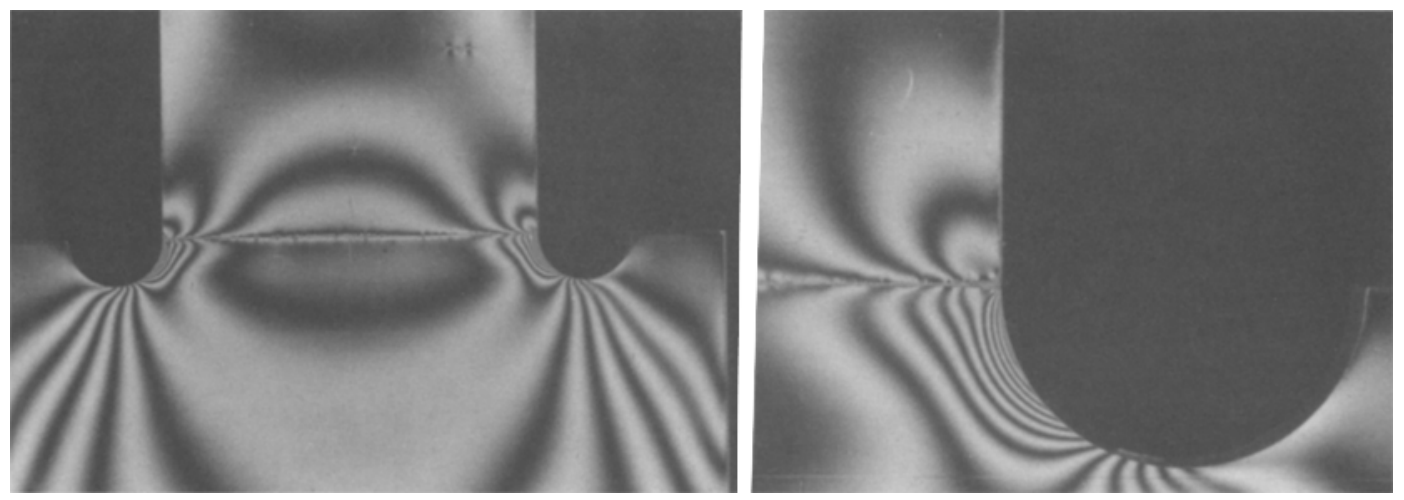

(a)
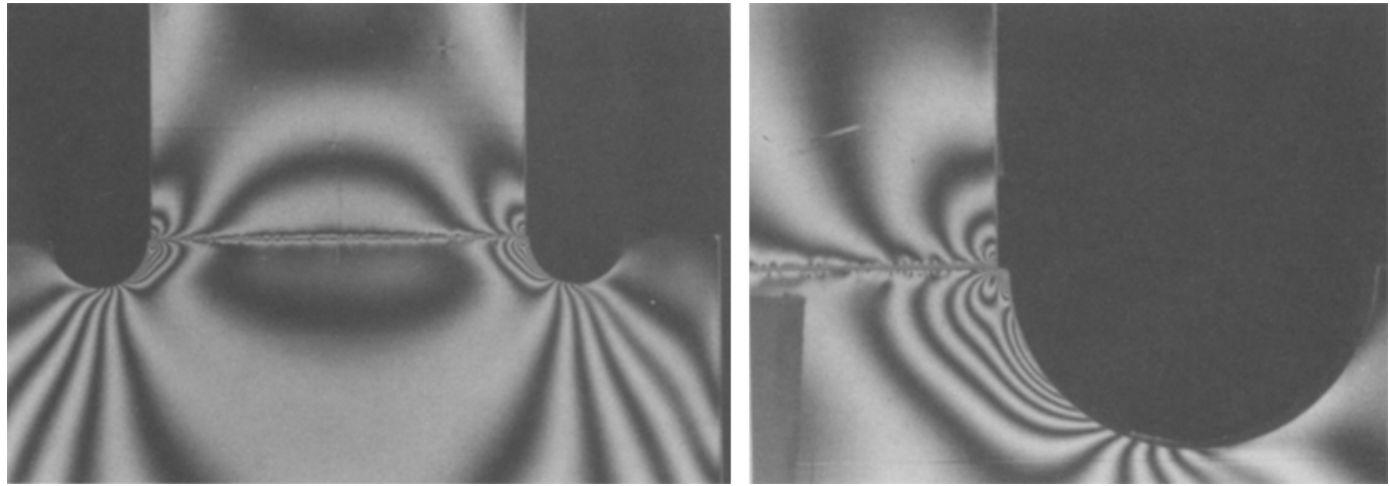

(b)
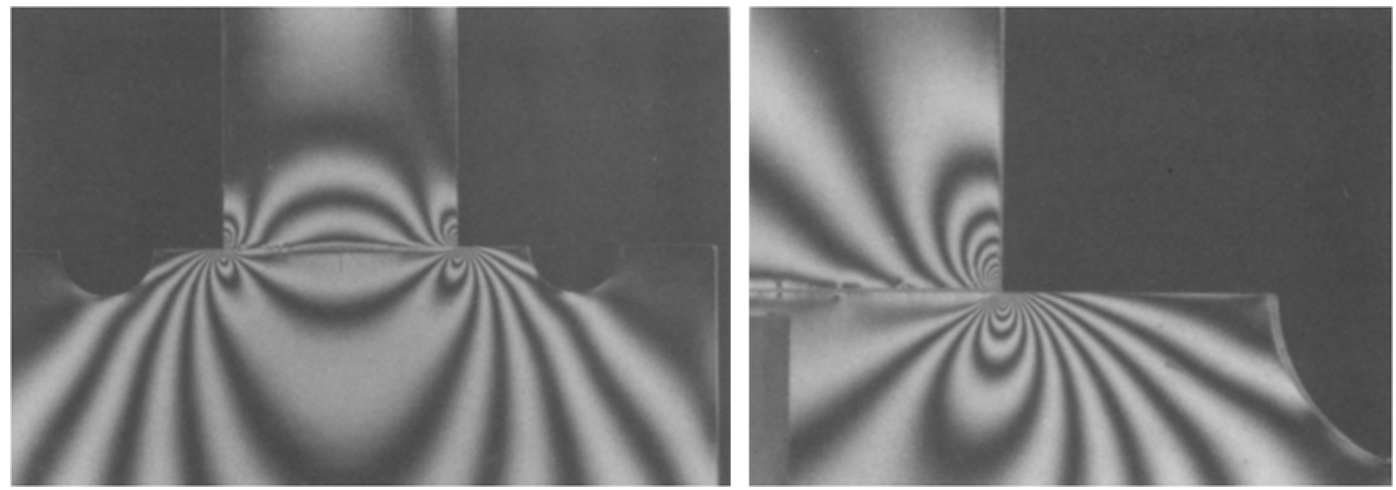

(c)

Fig. 8-Fringe patterns for the 'notch in the half-plane' case for $D=1.0 \mathrm{in}$. (a) $\zeta=0.5$, total picture and the enlargement of the right corner. (b) $\zeta=0.532$, total picture and the enlargement of the right corner. (c) $\zeta=1.252$, total picture and the enlargement of the right corner 
close proximity of the free surface of the notch [see sketch in Fig. 5(b) below]. A stress concentration is introduced at the notch root, however this may be preferable since the large stresses occur away from the contact surface.

The results of experiments for the case where the notches are placed on the half plane were analyzed in a similar manner to that for the notched indenters. The relation between $\ln$ (fringe order) and $\ln (\bar{r})$ was almost everywhere linear up to very small values of $r$. This finding, when compared to notched indenters, shows that the dominant region of the asymptotic form (1) is affected less by the presence of the notch in the half space than in the indenter. Further, in this case, a critical distance for the placement of the notches was found to be nonexistent. In contrast to notched indenters, this indicates the possibility of a continuous dependence of the singular field on the nondimensional distance $\zeta$.

The relations between the singularity power $p$ and nondimensional distance $\xi$ between the sharp corner of the indenter and the notch edge for different nondimensional notch diameters $\left(\frac{D}{a}\right)$ are shown in Figs. $6(a)$ and $6(\mathrm{~b})$.

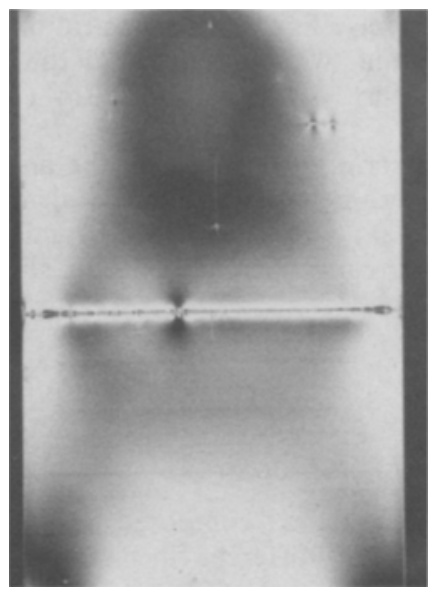

(a)

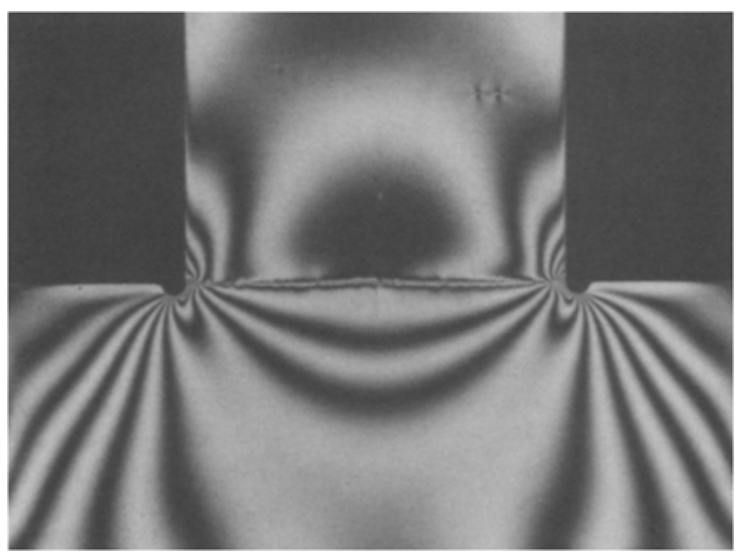

(b)

Fig. 9-Fringe patterns for the 'notch in the half-plane' case for (a) $D=\propto$ and (b) $D=0.250$ in. Average pressure $q=550$ psi. $\zeta=0.5$
The points indicate experimental findings, while the curves were plotted using the minimum square-root-deviation method. Generally $p$ decreases (the strength of the stress singularity increases) as a function of distance $\zeta$ but, more importantly, a nonsingular stress state at $\xi=0.5$ will exist only if the notch diameter is infinite and $\frac{L}{D}=$ 0.5 (the topmost experimental point corresponding to the 'infinite diameter' notch was obtained by compressing the indenter on an identical half space). In other words, the stresses in the right angle corner will be nonsingular only if two indenters of the same width and same material are in contact. For finite notch diameters, the stress field shows a weak singularity but is never nonsingular.

The pictures of fringe patterns for different cases are given in Figs. 7 through 9.

\section{Conclusions}

Following our earlier studies reported in Ref. 5, the present experimental investigations show that the stress singularity in the right angle corners of an indenter compressing a semi-infinite body can be controlled and totally eliminated by relief cuts (circular notches) along the free edges of the indenter. However, it is necessary to remember that the stress concentration in the notch root itself can reach a relatively high value in this case.

If the notch is cut out along the interface edge of the half-plane, the stress singularity in the corner can be reduced but never eliminated. In this latter case, the stress concentration at the notch root reaches a much smaller value than in the former case and usually does not need to be taken into account in strength calculations.

The practical applicability of the presented findings seems to be broad (e.g., the axisymmetric hole-pin contact problem) and important in engineering practice. A numerical simulation of the extension of the present problem to the axisymmetric hole-pin contact problem is currently being carried out. The results from that study will be presented elsewhere in the near future.

\section{Acknowledgment}

This work was supported by a grant from the Ford Motor Company, Scientific Research Laboratories. The authors acknowledge the constructive criticisms of the reviewers and appreciate their helpful comments.

\section{References}

1. Dundurs, J. and Lee, M.-S., "Stress Concentration at a Sharp Edge in Contact Problems," J. Elasticity, 2, 109 (1972).

2. Comninou, M., "Stress Singularity at a Sharp Edge in Contact Problems with Friction," J. Appl. Math. and Phys. (ZAMP), 27, 493 (1976).

3. Williams, M.L., "Stress Singularities from Various Boundary Conditions in Angular Corners of Plates in Extension," J. Appl. Mech., 19, 526 (1952).

4. Bogy, D.B., "Two Edge-bonded Elastic Wedges of Different Materials and Wedge Angles Under Surfaces Fractions," J. Appl. Mech., 38, 377 (1971).

5. Miniatt, E.C., Waas, A.M. and Anderson, WJ., "An Experimental Study of Stress Singularities at a Sharp Corner in a Contact Problem," EXPERIMENTAL MECHANICS, 30 (3), 281 (Sept. 1990).

6. Peterson, R.E., Stress Concentration Factors, John Wiley and Sons (1974).

7. Van Hulle, D., "Finite Element Analysis of Planar and Axisymmetric Rod-pin Contact Problem," Dept. of Aero. Eng. Rep. SM-91.3, Univ. of Michigan (199I).

8. Miniatt, E.C., "An Experimental and Numerical Study of Stress Relief at a Sharp Corner in a Contact Problem," Dept. of Aero. Eng. Rep. SM-90-3, Univ. of Michigan (1990). 